\title{
Le rôle des élites locales dans la consolidation du sentiment national
}

The Role of Local Elites in the Consolidation of National Identity

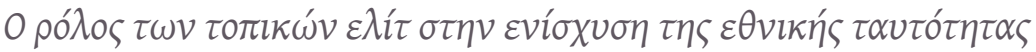

\section{Maria Zerva}

\section{OpenEdition} Journals

\section{Electronic version}

URL: https://journals.openedition.org/ceb/5819

DOI: $10.4000 /$ ceb.5819

ISSN: 2261-4184

\section{Publisher}

INALCO

\section{Printed version}

Date of publication: 1 March 2015

ISBN: 978-2-85831-224-5

ISSN: 0290-7402

\section{Electronic reference}

Maria Zerva, "Le rôle des élites locales dans la consolidation du sentiment national", Cahiers

balkaniques [Online], Hors-série | 2015, Online since 12 November 2015, connection on 06 July 2021.

URL: http://journals.openedition.org/ceb/5819; DOI: https://doi.org/10.4000/ceb.5819

This text was automatically generated on 6 July 2021.

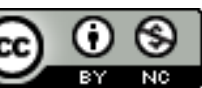

Cahiers balkaniques est mis à disposition selon les termes de la Licence Creative Commons Attribution - Pas d'Utilisation Commerciale 4.0 International. 


\title{
Le rôle des élites locales dans la consolidation du sentiment national
}

\author{
The Role of Local Elites in the Consolidation of National Identity

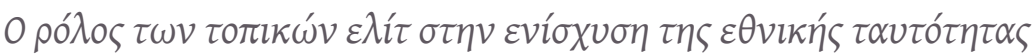

Maria Zerva

\section{Introduction}

1 Dans cet article, nous nous pencherons sur deux communautés (villages) grecques,

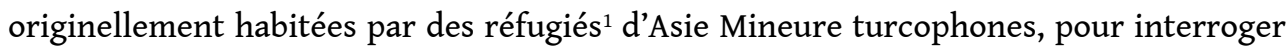
le rôle des élites locales dans la consolidation du sentiment national au sein de ces communautés. Pour ce faire, nous présenterons brièvement les deux villages et la recherche sur laquelle s'appuie cet article. Ensuite, il sera question de quelques résultats qui mettront en lumière la place du turc dans la vie et les représentations des membres de ces communautés. C'est à partir de ces résultats que nous tenterons de comparer ces localités et d'interpréter leurs éventuelles différences.

\section{Présentation de la recherche et contextualisation}

Il est question ici de deux villages grecs, fondés après l'échange des populations entre la Grèce et la Turquie prévu par le Traité de Lausanne en 1923, que nous nommerons, pour préserver l'anonymat des personnes concernées, village A et village B. Le village A est situé à proximité de Ioannina, au nord-ouest du pays, tandis que le village B se trouve dans le département de Serrès, au nord-est de la Grèce. La totalité des premiers habitants du village $B$ et une partie de ceux de A étaient originaires de la région de Bafra, ville du Pont occidental située en Turquie. Dans A, hormis ces personnes originaires du Pont occidental (Dytikopontii), furent également installées des personnes originaires de Cappadoce ainsi qu'un petit nombre d'Arméniens originaires aussi de cette région. Le dénominateur commun de ces personnes était la langue 
turque: aussi bien les Pontiques que les Cappadociens étaient turcophones et monolingues, au moment du déplacement forcé, comme le furent environ 100000 personnes parmi le 1,2 million de réfugiés recensés en 1928 .

Les réfugiés turcophones ont particulièrement mal vécu le déplacement vers cette nouvelle patrie, effectué sur la base de la religion qui était le critère de l'échange. Cet exode constitua un traumatisme profond pour tous les réfugiés et leurs descendants, non seulement en raison du déracinement, mais aussi en raison de l'accueil souvent hostile qu'ils reçurent, mais les turcophones orthodoxes durent, de surcroît, lutter contre des préjugés fondés sur une caractéristique qui les distinguait des autres réfugiés : la turcophonie. Celle-ci a été perçue comme un trait d'impureté ethnique (MARANTZIDIS, 2001, 90-91) qui enfreignait le modèle de la grécité, fondé sur la langue grecque et la religion chrétienne orthodoxe.

4 Le turc, perçu comme langue de l'ennemi, est ainsi jugé incompatible avec l'identité grecque que les populations en question revendiquaient pourtant. C'est pourquoi " gérer » l'usage du turc fut au cœur des stratégies mises en place par ces populations afin de pouvoir vivre leur identité grecque. Au centre de ces stratégies identitaires se trouvait l'abandon de la langue d'origine, c'est-à-dire l'assimilation linguistique. En outre, ces populations mettaient l'accent sur la religion comme repère identitaire, pratique déjà saillante dans l'Empire ottoman, où les peuples étaient différenciés par la religion, selon le système des millets. Ainsi, leur attachement à leur foi dépassait le sentiment religieux et signalait un acte identitaire (ibidem, 43-44).

Dans le cadre de nos recherches doctorales (ZERVA, 2011), nous avons rencontré des habitants des villages $\mathrm{A}$ et $\mathrm{B}$ et avons conduit des entretiens semi-directifs avec 36 d'entre eux : 23 entretiens ont été effectués dans le premier village et 13 dans le second, en A en 2005, et en B en 2007. Les entretiens ont été menés en grec et ont été transcrits minutieusement, représentant un volume d'environ 485 pages ( $922,5 \mathrm{~min}$ ). L'objectif de ce travail de recherche était de décrire le paysage sociolinguistique de ces communautés et d'y déterminer la place du turc. La description des dynamiques sociolinguistiques a été abordée au moyen de discours, de déclarations de nos interlocuteurs au cours des entretiens, ce qui a été déterminant sur le plan méthodologique, puisque les déclarations peuvent être en décalage par rapport aux pratiques. Ainsi avons-nous décidé de travailler avec la notion de représentations sociales $^{2}$, qui présente l'avantage d'insister sur le fait que l'humain ne détient pas la réalité objective, mais la construit, la réélabore en se la représentant.

\section{Quelques résultats sur les deux communautés}

6 Afin d'examiner le rôle des élites locales dans l'ancrage des représentations au sein de ces deux communautés, nous allons d'abord nous intéresser à la place du turc et aux représentations le concernant, pour pouvoir déceler les convergences et/ou divergences entre les deux villages.

\section{Assimilation linguistique}

7 En ce qui concerne l'assimilation linguistique, à savoir l'abandon de la langue d'origine au profit du grec, nous avons pu constater la restriction fonctionnelle du turc ainsi que la perte des compétences au fil des générations, bien que $80 \%$ de nos interlocuteurs 
aient une maîtrise solide du turc ${ }^{3}$. En outre, il est clair que le village B est plus avancé que A dans le processus d'assimilation linguistique, puisque les personnes interrogées maîtrisent peu le turc dès la $3^{e}$ génération, ce qui n'est nullement rencontré en $\mathrm{A}$, où toute la $3^{\mathrm{e}}$ génération le maîtrise bien.

\section{Transmission et représentations du turc}

De manière générale, le turc est minoré lorsqu'il est mis en discours : présenté comme une langue non choisie, qu'on subit malgré soi, extérieure à son identité, avec laquelle on prend ses distances, associée au passé ou à des expressions de gêne, d'hésitation et d'atténuation. La représentation du turc est, généralement, négative. En revanche, la mise en discours du grec est tout à fait différente; jamais minoré, il est, en effet, essentialisé et naturalisé dans l'identité de nos interlocuteurs, comme il l'est par ailleurs dans l'idéologie nationale, dont il constitue un élément fondamental.

9 Les turcophones orthodoxes adhèrent pleinement à cette idéologie et aux discours dominants ${ }^{4}$ qui y sont liés en les adoptant dans leur parole (cf. minoration du turc, majoration du grec) et dans leurs pratiques. Or, il semble qu'en $B$, l'ancrage des discours dominants soit plus important qu'en A : non seulement les représentations du turc y sont plus négatives, mais on y est aussi plus enclin à favoriser l'abandon du turc (ZERVA, 2011 3 ,330-331, 344, et passim). Cela paraît très clairement dans la transmission du turc aux enfants. Lorsqu'on pose la question de savoir si le turc a été transmis aux enfants, la réponse est plus souvent non que oui ${ }^{6}$. Cette tendance est plus forte en B. Il est par ailleurs intéressant que les témoins qui n'avaient pas d'enfants au moment de l'enquête et qui ont, par conséquent, exprimé seulement leur intention ou non de transmettre le turc, soient nettement plus nombreux à répondre positivement en $\mathrm{A}$ qu'en $B^{7}$. Sans pouvoir rentrer dans les détails, soulignons que le processus de l'assimilation linguistique est plus avancé en B qu'en A également sur le plan des représentations. Dans le village A, on tient davantage au turc, bien que ce processus soit clairement engagé, et les témoins de ce village adoptent plus souvent une attitude ${ }^{8}$ positive envers la transmission, même si cela ne correspond pas à leurs pratiques (ibid., 388-439).

\section{Musique}

10 Le décalage des représentations entre les deux villages devient plus clair dans le cas de leur production musicale, telle qu'elle a été « officialisée » avec la sortie d'un CD de la part de chacune de ces communes qui ont adopté des stratégies différentes. En A, on a sorti un CD intitulé Danses et chansons traditionnelles du village A de Ioannina, édité par l'association culturelle locale, et l'Association Internationale d'Art populaire, dans lequel toutes les chansons sont en turc, tandis que les mélodies reflètent la composition ethnique mixte du village dans les années 1920 : aux mélodies pontiques s'ajoutent des mélodies typiques de la région de Cappadoce. D'un autre côté, le CD de B ${ }^{9}$ ne comprend que des chansons en mélodie pontique, ce qui est normal si l'on songe à la composition ethnique des premiers habitants du village, uniquement pontiques. À une exception près : une chanson se rapproche de par sa mélodie de celles du reste de l'Asie Mineure (il s'agit du très connu Koniali qu'on retrouve un peu partout en Asie Mineure et en Grèce de l'Est), mais reste tout de même proche du style pontique, grâce notamment aux instruments utilisés pour la jouer. La langue des douze chansons sur les quatorze 
du CD est le grec pontique. Les deux dernières sont en turc. C'est pendant notre séjour à $\mathrm{B}$, au cours de l'enquête, que nous avons par ailleurs appris que les paroles en grec pontique étaient le résultat d'une traduction depuis le turc (ZERVA, 2011, vol. II, 362 et 488).

Il va de soi que ce choix de présenter un $\mathrm{CD}$ avec la musique du village en grec pontique et non en turc est significatif. Les raisons de cette traduction sont développées par un de nos interlocuteurs, $\mathrm{Bm} 36^{10}$. Elle serait le résultat de deux traductions consécutives, du turc au grec moderne et du grec moderne au grec pontique :

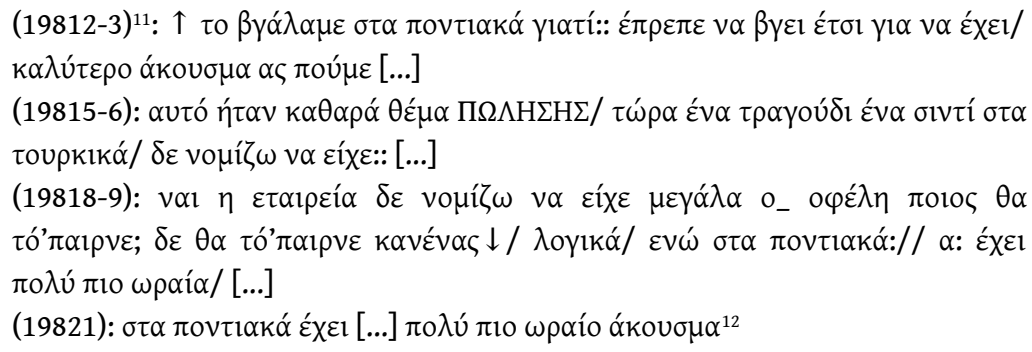

Pour ce témoin, il s'agit d'un choix soumis à la fois à une logique marchande et à un parti-pris esthétique, dont le turc ressort minoré. En revanche, le grec pontique est majoré, ce qui est certainement lié au contexte géographique du village, situé en Macédoine grecque, où une grande partie de réfugiés d'Asie Mineure et partant, de Pontiques a été installée. L'élément pontique y est donc très important. Il convient également de noter que les Pontiques sont, d'une manière générale, le groupe de réfugiés grecs le plus dynamique et le mieux organisé, avec un grand nombre d'associations à travers la Grèce (VERGETI, 1994) qui animent souvent des groupes de danses pontiques. Il semblerait donc qu'il existe réellement un marché de musique pontique en Grèce. Ainsi, les propos de Bm36 apparaissent tout à fait cohérents.

Rappelons aussi que le village $B$ a été fondé et initialement habité par des personnes originaires de la région de Pafra/Bafra du Pont occidental, donc des Pontiques turcophones, contrairement à $\mathrm{A}$ dont la population initiale était mixte. Les Bafralides revendiquent leur identité pontique, qui ne leur a pas toujours été volontiers reconnue par les Pontiques grécophones ${ }^{13}$. En publiant un CD de leur musique traditionnelle majoritairement en grec pontique, ils envoient un message multiple : ils appartiennent à la communauté pontique (et, bien sûr, grecque), ils rejettent la langue turque comme extérieure à leur identité et ils adhèrent aux discours dominants selon lesquels le Grec - mais aussi le Pontique -, par excellence est grécophone, et les reconnaissent même si ou parce qu'ils s'y opposent $t^{14}$.

\section{Souffrances subies et histoire-mythe}

14 Les questions déjà abordées, et notamment celle de la musique, révèlent les quelques divergences des deux villages en ce qui concerne les représentations et l'ancrage des discours dominants - divergences qui se limitent pourtant à des nuances puisque l'adhésion aux discours dominants et à l'idéologie dominante est certaine dans les deux cas. Nous compléterons ce tableau avec deux derniers points qui concernent le passé de ces populations sur le territoire ottoman. 

réponses données, une grande partie de nos interlocuteurs a fait référence aux souffrances infligées à leurs ancêtres par les Turcs. Si ces souffrances reviennent souvent dans le discours des témoins ( $52,8 \%$ les évoquent), elles sont nettement plus présentes dans le discours des habitants de B (92,3\%) qu'en A $(30,4 \%)$. Le contexte historique nous fournit des éléments d'explication: comme mentionné, les réfugiés installés en B étaient originaires du Pont occidental, fief de la guérilla contre les Ottomans et lieu de graves persécutions entre 1914 et 1922 (VERGETI, 1994, 102-124). C'est pourquoi la mémoire des multiples souffrances subies est toujours vivante chez la quasi-totalité des témoins. En revanche, en $\mathrm{A}$, où les réfugiés venaient aussi de l'intérieur d'Asie Mineure (Cappadoce), peu touché par la guerre, la mémoire des souffrances est moins présente.

il est vrai que la mémoire joue un rôle important dans la construction des représentations, il n'en reste pas moins vrai qu'elle répond essentiellement aux besoins du présent. Ainsi, la mémoire des souffrances subies est encouragée par les discours dominants et l'idéologie nationale, dans le cadre de la construction d'une identité grecque. Cela ne veut pas dire que les ancêtres des témoins n'aient pas souffert et subi de persécutions. Mais il semble légitime de penser que la mémoire personnelle ait été alimentée et encouragée par la mémoire officielle qui met l'accent sur certains aspects du confit gréco-turc et en omet d'autres. Le discours martyrologique et/ou héroïque constitue d'ailleurs un moyen courant de légitimer l'appartenance à la nation (SIDERI, 2008).

rôle des élites locales dans cette réappropriation de la mémoire doit être déterminant, comme l'atteste d'ailleurs GEFOU-MADIANOU à propos des Arvanites de Mesogée $(1999,423)$. Il en va de même dans le cas qui nous intéresse, en ce qui concerne l'explication de la turcophonie sur la base d'un récit qui se situe entre mythe et histoire ${ }^{15}$, selon lequel les Grecs turcophones ont été confrontés à un cruel dilemme imposé par les Ottomans ; ils devaient choisir entre leur langue (le grec) et la religion, et ont finalement opté pour la religion, considérée comme la base de l'identité grecque. Selon MARANTZIDIs, les élites centrales et locales ont certainement joué un rôle important dans la création et la diffusion de l'histoire-mythe, étant donné qu'elle incarne parfaitement l'idéologie nationale (2001, 47-48).

18 Cela apparaît d'ailleurs dans les propos d'un témoin du village A. L'anecdote qu'il raconte montre que l'histoire-mythe relèverait plus du savoir que de la mémoire. Notre hypothèse est que l'homme dont il est question a participé à l'élaboration du $\mathrm{CD}$, faisant lui-même partie de l'Organisation internationale d'Art populaire. Il appartiendrait ainsi à une élite ni centrale ni locale, mais intellectuelle, qui a contribué à la diffusion d'une explication politiquement correcte de la turcophonie ${ }^{16}$.

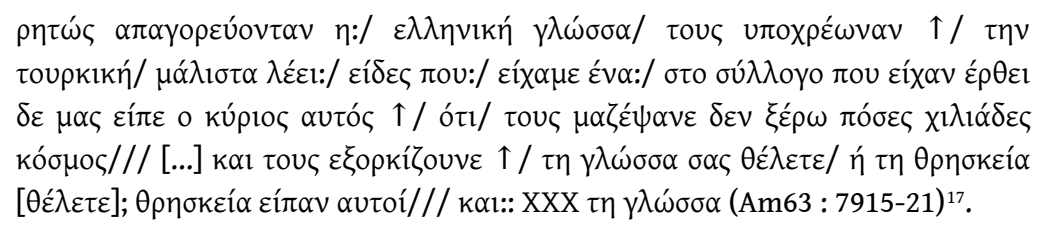

Dans tous les cas, l'histoire-mythe revient fréquemment dans notre corpus, mais pas toujours dans cette version "canonique»; le récit est souvent confus, parsemé d'incohérences et de contradictions. Or, il convient de noter qu'il apparaît plus souvent 
dans cette version "canonique» dans la bouche des habitants de $\mathrm{B}$ qu'en $\mathrm{A}^{18}$. Plus généralement, l'usage de la langue turque par les ancêtres est très souvent expliqué par des persécutions infligées par les Ottomans et ce, de nouveau, plus souvent en B qu'en $\mathrm{A}^{19}$. Il est clair que le discours martyrologique, les persécutions et les souffrances subies sont utilisées dans une visée argumentative, pour justifier la turcophonie et légitimer l'appartenance à la nation grecque.

\section{Réflexions sur ces résultats et rôle des élites locales}

Si les deux communautés adhèrent pleinement aux discours dominants et à l'idéologie nationale, il existe de légères différences en ce qui concerne l'ancrage de ces derniers et les représentations. Il est vrai que le contexte sociohistorique peut fournir des éléments d'explication. Pour ce qui est des souffrances et des persécutions, nous avons vu que la population de $\mathrm{B}$, originaire du Pont occidental, a gardé en mémoire la guérilla menée sur le territoire ottoman et les persécutions subies. La mémoire d'un passé héroïque n'a pas été inintéressante pour la construction de l'identité nationale, c'est pourquoi le discours martyrologique a trouvé racine dans les deux communautés, bien qu'il soit moins présent en $A$.

21 Le village $B$ est d'ailleurs situé en Macédoine grecque, terre à forte concentration de réfugiés, notamment pontiques. La composition ethnique initiale homogène, mais aussi le contact intense avec d'autres réfugiés pontiques ont dû renforcer la présence de l'élément pontique dans la construction identitaire de ses habitants. Or, il ne faut pas oublier que l'identité pontique est un " gage » de grécité et il semblerait qu'il s'agit d'un renforcement en partie choisi (cf. le choix de traduire les chansons traditionnelles en grec pontique ${ }^{20}$ ) suivant une stratégie identitaire d'adaptation aux normes de la grécité. Ces normes ont été d'ailleurs pendant longtemps dictées par les valeurs conservatrices en Grèce. Il nous semble opportun de risquer ici une autre interprétation, selon laquelle, en $B$, on a adhéré de manière plus engagée aux valeurs nationales aussi en raison des récompenses et de la protection que la structure étatique aurait offertes à ceux qui se sont rangés à droite pendant la guerre civile ${ }^{21}$. Parmi eux figuraient les Pontiques turcophones qui ont de cette manière aussi (entre autres) gagné leur place dans la nation.

Cette place a été revendiquée par les populations et leurs élites comme un choix identitaire, mais aussi pragmatique, le seul à leur assurer de bonnes conditions d'insertion sociale. Il s'agit là d'un des principaux objectifs des élites locales : assurer à leurs compatriotes une insertion dans l'ensemble national dans les meilleures conditions possibles (cf. KARAKASIDOU, 199722 ; PANOPOULOS, 2006 ; GEFOU-MADIANOU, 1999). Ce but a été poursuivi par les élites locales des villages en question, qui peuvent être définies grossièrement comme les personnes ou les familles qui disposent des biens (matériels, culturels...) et/ou des pouvoirs (politiques, symboliques...) à un degré maximal (MARANTZIDIS, 2001, 153-154 et 65).

Dans notre cas, deux personnes se sont distinguées comme membres de l'élite locale, Am49, en ce qui concerne le village A, et Bm81 pour ce qui est du village B. Am49 était depuis de nombreuses années le président du conseil communal et de l'association culturelle du village, et il a laissé entendre que sa famille en général exerçait souvent des mandats politiques ${ }^{23}$. Personne reconnue et influente dans la communauté, il a organisé la publication du $\mathrm{CD}$ de musique qui, nous le rappelons, est sorti entièrement 
en turc. En même temps, notons que, parlant de la turcophonie, il ne l'a pas liée à des souffrances subies et nous a livré aussi bien la version "canonique " de l'histoiremythe qu'une version plus rationnelle, à savoir qu'il était normal que les gens adoptent le turc, puisqu'ils vivaient dans un environnement turcophone (l'administration, le commerce, etc. utilisaient le turc). Il s'est montré par ailleurs positif envers le turc qu'il chérit et il aurait aimé que ses enfants le parlent, parce qu'il est lié à leurs racines (il ne le leur a pourtant pas transmis).

D'un autre côté, la personne que nous considérons comme éminent représentant de l'élite locale au village B est Bm81, érudit local qui s'est distingué tant par ses études remarquables ${ }^{24}$ que par les postes qu'il a occupés (instituteur au village, puis, après des études de droit, haut fonctionnaire à Thessalonique). Il constitue une référence incontournable en ce qui concerne les affaires du village, notamment pour tout ce qui a trait à l'histoire et à la culture : toutes les personnes que nous avons rencontrées nous ont renvoyée vers lui pour des « renseignements plus complets ». En même temps, nous savons que c'est lui qui donne des instructions concernant les commentaires historiques qu'on doit faire lors de représentations des danses et des traditions du village. En outre, il tient des archives avec des témoignages des premiers réfugiés qu'il a lui-même traduits du turc, comme il l'a d'ailleurs fait pour les chansons du CD dont il a été question. Au cours de son entretien, il est devenu clair qu'il était fervent défenseur de l'idéologie nationale. Il a retracé l'histoire de Pafra/Bafra depuis l'Antiquité jusqu'au début du $\mathrm{xx}^{e}$ siècle et l'échange des populations, et a beaucoup insisté sur les combats des guérilleros pontiques, mais aussi sur les persécutions et les souffrances subies par les populations. Il est intéressant de noter qu'il nous livre une version "canonique » de l'histoire-mythe, mais qui cesse de l'être dès lors que Bm81 surenchérit sur les persécutions et raconte que, malgré le dilemme entre langue et religion et malgré le choix opéré (de garder la religion et d'abandonner la langue grecque), finalement la religion aussi a été interdite. En ce qui concerne le turc, il se montre indifférent, si ce n'est négatif, et il est dans tous les cas positif envers l'assimilation linguistique.

Il ressort donc clairement que les deux hommes sont très bien intégrés dans les mécanismes étatiques, mais ont des orientations idéologiques personnelles différentes. Celles-ci doivent avoir été influencées, entre autres, aussi par leur âge : plus on est jeune, mieux on est intégré et plus on est prêt à revendiquer sa différence (cf. GEFOU-MADIANOU, 1999). Le contexte géographique et sociohistorique doit avoir également joué un rôle, comme nous l'avons expliqué ci-dessus. Dans tous les cas, il nous semble que ces différences se sont répercutées dans les représentations et dans l'ancrage des discours dominants de chaque village qui seraient ainsi en partie expliqués par le rôle des élites locales. 


\section{BIBLIOGRAPHY}

ELOEVA Fatima, 1998, « Les Grecs turcophones de Géorgie. Territoires et tradition orale à Tsalka et Tetritskaro », in M. BRUNEAU (dir.). Les Grecs pontiques. Diaspora, identité, territoires, Paris : CNRS éditions, p. 137-141.

GEFOU-MADIANOU Dimitra, 1999, “Cultural Polyphony and Identity Formation: Negotiating Tradition in Attica", American Ethnologist, vol. 26, no 2: 412-439.

JODELET Denise, 1989, « Représentations sociales : un domaine en expansion », in Denise JODELET (dir.), les Représentations sociales, Paris : Presses Universitaires de France, p. 31-61.

KARAKASIDOU Anastasia, 1997, Fields of wheat, hills of blood, Passages to Nationhood in Greek Macedonia 1870-1990, Chicago: The University of Chicago Press.

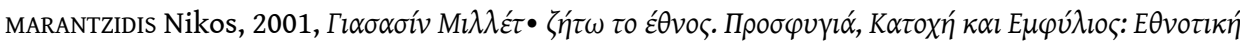

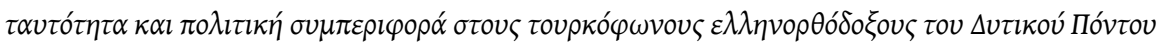
[Yasassin Millet. Vive la nation. Réfugiés, occupation et guerre civile : identité ethnique et conduite politique des turcophones grecs orthodoxes du Pont occidental], Héraklion : Presses Universitaires de Crète.

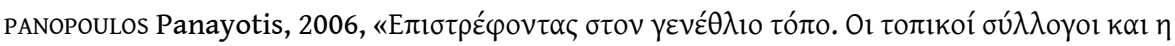

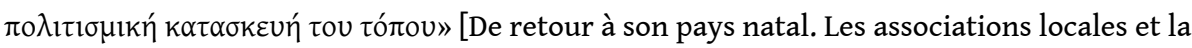

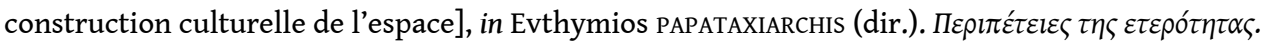

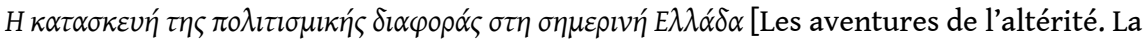
construction de la différence culturelle dans la Grèce d'aujourd'hui], Athènes : Alexandria, p. 87-103.

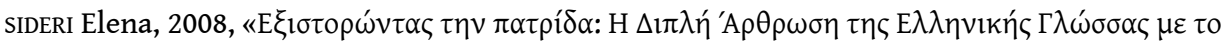

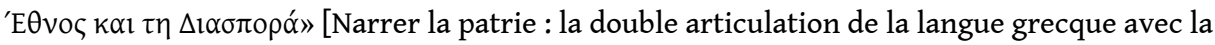
nation et la diaspora], Studies in Greek Linguistics, Language and Society, Thessalonique : Institut des études néohelléniques, Université Aristote.

VERGETI Maria, (2000/1994), A T $\alpha v \tau o ́ \tau \eta \tau \alpha \varsigma$ [Du Pont en Grèce. Processus de formation d'une identité ethno-régionale], Thessalonique : Kyriakidis.

ZERVA Maria, 2011, Les Grecs turcophones orthodoxes : une étude sociolinguistique, Thèse de doctorat (inédite), Université de Strasbourg, vol. I et vol. II.

\section{NOTES}

1. Réfugiés est le nom utilisé pour désigner aussi bien les réfugiés proprement dits, à ceux qui fuirent, en 1922, l'Anatolie, que les «personnes échangées » ( $\left.\alpha v \tau \alpha \lambda \lambda \alpha \dot{\xi} \xi_{\imath \mu \nu l}\right)$ qui furent forcées à quitter la Turquie suite au Traité de Lausanne. En Grèce, le nom est fréquemment utilisé pour désigner également les descendants des réfugiés, et ce jusqu'à la $4^{e}$ génération. 
2. Selon JODELET et sa définition de la représentation, celle-ci «est une forme de connaissance, socialement élaborée et partagée, ayant une visée pratique et concourant à la construction d'une réalité commune à un ensemble social » $(1989,36)$.

3. L'écrasante majorité des personnes maitrisant bien le turc appartient aux $2^{\mathrm{e}}$ et $3^{\mathrm{e}}$ générations des réfugiés qui constituent d'ailleurs la majorité de nos interlocuteurs (78\% des personnes interrogées appartiennent aux $2^{\mathrm{e}}$ et $3^{\mathrm{e}}$ générations, tandis que seuls $22 \%$ appartiennent à la $4^{\mathrm{e}}$ génération). Or, bien que l'assimilation linguistique soit avancée, il convient de remarquer qu'elle n'a pas été accomplie avec la 4 e génération en ce qui concerne $\mathrm{A}$, et notamment les individus les plus âgés de cette génération, nés fin des années 1960. En revanche, les individus nés à partir de 1975 ont généralement une maitrise faible ou nulle du turc, à quelques exceptions près. À $B$, la $4^{e}$ génération n'a aucune connaissance du turc, mais il faut signaler que nous n'avons rencontré que 2 personnes de cette génération (sur les 13 à $\mathrm{B}$ ). L'affaiblissement de la maîtrise du turc est visible dès la $3^{\mathrm{e}}$ génération, ce qui nous permet de présumer que l'assimilation y est véritablement plus avancée (ZERVA, 2011, 142-272 et 508-512).

4. Par discours dominants, nous entendons des ensembles de discours culturellement partagés qui circulent dans une société, alimentent et, en même temps, contraignent la production discursive locale des sujets (le discours avec minuscule) (cf. KIESLING, 2006, 261-265). Ces discours sont étroitement liés à l'idéologie/aux idéologies dominantes.

5. Lorsque la référence ZERVA 2011 n'est accompagnée d'aucune précision, il s'agit du vol. I, à savoir du corps de la thèse.

6. Même si leur non a été démenti par la suite par l'entretien de leurs propres enfants. En général, l'ampleur de la transmission du turc a été minorée, ce qui ressort clairement de la confrontation des réponses sur la transmission de la $2^{\mathrm{e}}$ génération et de la maîtrise du turc de la $3^{\mathrm{e}}$ génération et, de manière similaire, de la comparaison entre les réponses de la $3^{\mathrm{e}}$ et de la $4^{\mathrm{e}}$ génération.

7. $75 \%$ des témoins de B n'ayant pas d'enfants répondent qu'ils ne transmettront pas le turc, contre $20 \%$ en A. Inversement, $80 \%$ des témoins en A n'ayant pas d'enfants expriment leur intention de transmettre même le peu de turc qu'ils connaissent à leurs futurs enfants contre $25 \%$ en B.

8. Dans l'analyse de nos données sur la transmission, nous avons fait une distinction subtile entre représentation et attitude. Dans ce cas, la notion de représentation a servi pour tout ce qui touchait à la mise en discours du sujet de la transmission (effective ou intentionnelle dans le cas où la personne n'avait pas d'enfants), à son développement et aux commentaires qui l'accompagnaient. En revanche, la notion d'attitude a servi pour désigner la position négative ou positive de nos interlocuteurs à l'égard de la transmission, indépendamment de la transmission effective.

9. Nous avons pris connaissance de ce $\mathrm{CD}$ au cours de notre enquête et n'avons pu nous procurer qu'une copie qu'un de nos interlocuteurs a gravée pour nous, c'est pourquoi nous ne disposons pas d'informations relatives à l'édition.

10. Voici, sommairement, les conventions de transcription: Bm36, homme $(m) d u$ village B (B) âgé de 36 ans (36) au moment de l'entretien. /, //, /// pause plus ou moins longue. :, ::, ::: allongement plus ou moins long de la voyelle ou de la consonne qui précède. $=$ reprise de discours interrompu. $\uparrow$ intonation ascendante. $\downarrow$ int. descendante. ; int. ascend. interrogative) commentaire du chercheur sur la qualité de la voix ou l'environnement. [...] énoncé pour lequel nous ne sommes pas sûres, mais 
faisons une proposition d'écoute. XXX énoncé inintelligible. Les Majuscules marquent le début des noms propres ou, lorsqu'elles sont appliquées à un mot ou à des lettres d'un mot, dénotent une accentuation emphatique ou bien une voix plus forte que pour le reste de l'énoncé. Le signe [...] indique l'absence du tour de parole de l'enquêteur pour des raisons d'espace. L'intervention de l'enquêteur est ici minimale et se contente de réalimenter la discussion.

11. Les chiffres entre parenthèses renvoient à la numérotation automatique des lignes de notre corpus (ZERVA, 2011, vol. II).

12. Voici une traduction qui suit de près le texte grec ainsi que les hésitations, l'intonation et les ratés du locuteur: $\uparrow$ nous l'avons sorti en grec pontique parce que:: il fallait que ça sorte comme ça pour avoir/ un meilleur résultat quoi [...] ça c'était purement une question de VENTE/ maintenant une chanson un CD en turc/ je ne pense pas qu'il aurai::t [...] en grec pontique [...] le résultat est beaucoup mieux [...] oui la maison (des disques) je ne pense pas qu'elle ferait un grand bénéfice qui l'achèterait; personne ne l'achèterait $\downarrow /$ logiquement/ tandis qu'en grec pontique :// ah, il a un beaucoup plus joli/ (résultat).

13. Il semble que les Pontiques grécophones ont un sentiment de supériorité envers les Pontiques turcophones. Cela ressort des propos de certains de nos interlocuteurs, mais aussi d'une discussion informelle avec Nikos Marantzidis ainsi que d'observations que nous avons faites nous-même lorsque nous avons fréquenté pendant trois ans une

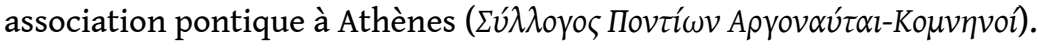

14. Ajoutons également que l'élément pontique dans la musique traditionnelle du village B semble être renforcé ou construit rétrospectivement selon une logique identitaire - rappelons que les Pontiques sont acceptés comme Grecs par la communauté grecque sans réserve -, tandis que leur spécificité "ethno-locale », selon VERGETI (1994), est valorisée. Ainsi, la distinction entre la musique de B et la musique pontique est perçue par les personnes les plus âgées, tandis que la conscience de cette distinction fait défaut chez les plus jeunes, qui identifient la musique de leur village à la musique pontique. Pour plus de détails, voir ZERVA, 2011, 375-380.

15. Nous empruntons le terme histoire-mythe à MARANTZIDIS qui l'utilise, car sa véracité n'est pas prouvée et en même temps importe peu, puisque ce qui compte est le fait que cette narration ait influencé la manière dont ces populations percevaient la réalité et a répondu aux besoins psychologiques des populations en expliquant l'inexplicable, en justifiant et en gérant la contradiction entre langue turque et identité grecque (2001, 46-48). eloeva d'ailleurs met en question la véracité de ce récit, selon elle très improbable dans le contexte ottoman $(1998,139)$.

16. Les deux membres de cette Organisation ont d'ailleurs signé un texte introductif dans le livret qui accompagne le $\mathrm{CD}$, intitulé «La tradition de musique et de danse du village A », dans lequel on retrouve l'histoire-mythe.

17. la:/ la langue grecque/ était formellement interdite/ on les obligeait $\uparrow$ / (à parler) turc/ et même::/ tu vois qua:nd/ nous avions u:n/ lorsqu'ils sont venus à l'association ce Monsieur ne nous a pas dit $\uparrow$ / que/ on les a rassemblés je ne sais pas combien de milliers de gens///[...] et on les adjure $\uparrow /$ vous voulez votre langue/ ou [vous voulez] votre religion; eux ils ont dit la religion/// e::t XXX la langue.

18. À B, 7 témoins sur 13 l'évoquent (53,85\%), contre 7 sur 23 à A (30,43\%).

19. À $B$, ils sont 12 sur 13 à expliquer ainsi la turcophonie (92,3\%), contre 13 sur 23 à $\mathrm{A}(56,52 \%)$. 
20. Nous ne pouvons pas développer ici, mais il est intéressant de noter que les habitants du village B se désignent dans leur majorité en tant que "Bafralides" (originaires de Bafra/Pafra), sauf Bm81 (voir ci-dessous) et son entourage familial, également interviewé, qui se disent Pontiques.

21. La facette ethnique de la guerre civile en Macédoine est traitée de manière convaincante par MARANTZIDIS (2001, voir en particulier p. 152-155).

22. Selon KARAKASIDOU, l'élite locale a contribué à l'hellénisation de la ville d'Assiros étudiée.

23. Il nous a parlé d'un adage qui circulait dans le village avant les élections municipales « pourvu que l'élu, quel qu'il soit, soit quelqu'un de la famille X (son nom

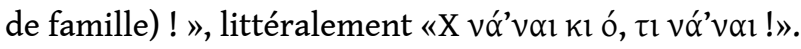

24. D'autant plus qu'il est né en 1926 dans un environnement très pauvre.

\section{ABSTRACTS}

This paper examines the role of local elites in the consolidation of national identity through the case study of two Greek villages, originally inhabited by Turkish speaking Asia Minor refugees. Greek national identity is irrefutable in both cases, yet there are some differences between them which can be explained by socio-historical and geographical context as well as by the specific role of local elites.

Cet article interroge le rôle des élites locales dans la consolidation du sentiment national dans deux communautés en Grèce, initialement habitées par des réfugiés d'Asie Mineure qui, lors de leur déplacement forcé, étaient turcophones. Si l'intégration de la communauté nationale est certaine dans les deux cas, il semble que les quelques différences constatées puisent leur source aussi bien dans le contexte sociohistorique et géographique des communautés que dans le rôle de leurs élites locales.

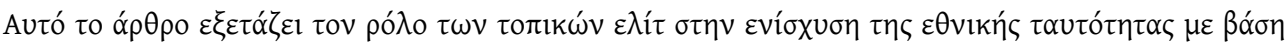

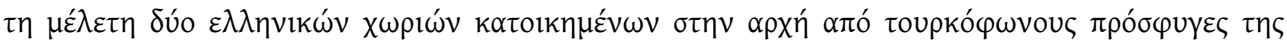

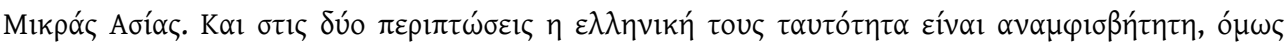

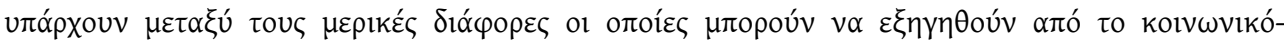

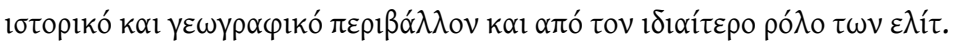


INDEX

Geographical index: Grèce

motsclestr Ulusal kimlik, Mülteciler, Türkçe konuşan Rumlar, Asimilasyon, Yunanistan, Yirminci Yüzyıl, Zihniyetlerin Tarihi, Toplumsal Tarih

motsclesmk НАЦИОНАЛНИОТ ИДЕНТИТЕТ, БЕГАЛЦИ, ТУРСКИ ЈАЗИК ГРЦИТЕ, АСИМИЛАЦИЈА, ГРЦИЈА, ДВАЕСЕТТИОТ ВЕК, ИСТОРИЈА НА МЕНТАЛИТЕТОТ, СОЦИЈАЛНА ИСТОРИЈА

Keywords: national identity, refugees, Turkspeaking Greeks, assimilation, Greece, Twentieth century, history of mentalities, social history

Mots-clés: identité nationale, réfugiés, Grecs turcophones, assimilation

Subjects: Histoire des mentalités, Histoire sociale

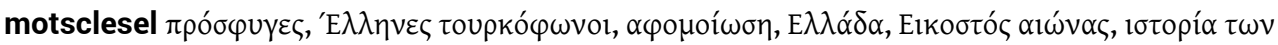

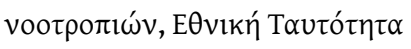

Chronological index: vingtième siècle

\section{AUTHOR}

MARIA ZERVA

Université de Strasbourg 